\title{
The stress polarity signaling (SPS) pathway serves as a marker and a target in the leaky gut barrier: implications in aging and cancer
}

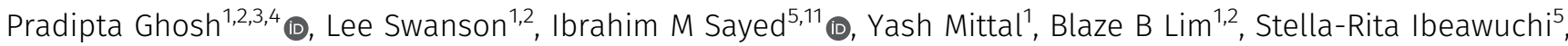

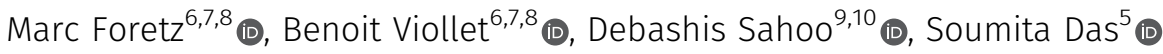

The gut barrier separates trillions of microbes from the largest immune system in the body; when compromised, a "leaky" gut barrier fuels systemic inflammation, which hastens the progression of chronic diseases. Strategies to detect and repair the leaky gut barrier remain urgent and unmet needs. Recently, a stress-polarity signaling (SPS) pathway has been described in which the metabolic sensor, AMP-kinase acts via its effector, GIV (also known as Girdin) to augment epithelial polarity exclusively under energetic stress and suppresses tumor formation. Using murine and human colon-derived organoids, and enteroid-derived monolayers (EDMs) that are exposed to stressors, we reveal that the SPS-pathway is active in the intestinal epithelium and requires a catalytically active AMP-kinase. Its pharmacologic augmentation resists stress-induced collapse of the epithelium when challenged with microbes or microbial products. In addition, the SPS-pathway is suppressed in the aging gut, and its reactivation in enteroid-derived monolayers reverses aging-associated inflammation and loss of barrier function. It is also silenced during progression of colorectal cancers. These findings reveal the importance of the SPS-pathway in the gut and highlights its therapeutic potential for treating gut barrier dysfunction in aging, cancer, and dysbiosis.

DOI 10.26508/Isa.201900481 | Received 10 July 2019 | Revised 21 January 2020 | Accepted 22 January 2020 | Published online 10 February 2020

\section{Introduction}

The gut is a complex environment; the gut mucosa maintains immune homeostasis in physiology by serving as a barrier that restricts access of trillions of microbes, diverse microbial products, food antigens, and toxins to the largest immune system in the body. The intestinal barrier is the largest mucosal surface that separates diverse stressors (trillions of microbes, toxins, and food antigens) on one side from the largest immune system on the other. A compromised gut barrier allows microbes and unwanted antigens to cross the epithelium and generate inflammation (systemic endotoxemia), which may contribute to a variety of diseases, ranging from metabolic syndrome and chronic organ dysfunctions to neurodegenerative diseases and cancers (Yacyshyn et al, 1996; Barbara, 2006; Camilleri \& Gorman, 2007; Sandek et al, 2007, 2008, 2012; Alam et al, 2014; Bischoff et al, 2014; Nouri et al, 2014; Samsam et al, 2014; van De Sande et al, 2014; Clairembault et al, 2015; Lee et al, 2015; Buscarinu et al, 2016; Xue et al, 2016; Ghosh, 2017). Evidence also shows that aging-related genes, that is, the sirtuins (SIRTs1, 3, 6), are critical for the integrity of the gut barrier and for controlling inflammation in the gut (Akimova et al, 2014; Akbulut et al, 2015; Liu et al, 2017b; Zhang et al, 2018). Despite the traction and the discovery of plausible targets to strengthen the barrier, for example, myosin light-chain kinase (Cunningham \& Turner, 2012), our knowledge of the underlying mechanism(s) that reinforce the barrier when faced with stressors is incomplete, and practical strategies for pharmacologic modulation of the gut barrier remains unrealized.

The primary factor preventing the free access of stressors to our immune cells is a single layer of polarized intestinal epithelial cells strung together in solidarity by cell-cell junctions. Loss of cell polarity and junctions not only impairs organ development and function but can also serve as one of the first triggers for oncogenesis (Martin-Belmonte \& Perez-Moreno, 2012). Establishment, maintenance (at baseline), and augmentation (upon stress) of epithelial barriers are achieved by signaling pathways that regulate polarization of epithelial cells. Epithelial polarity is established and maintained by a set of evolutionarily conserved signaling pathways whose integration in space and time dictates overall epithelial morphogenesis (St Johnston \& Sanson, 2011), for example, CDC42 and PAR proteins, such as the PAR3-PAR6-aPKC polarity complex (Wodarz \& Nathke, 2007) and pathways that regulate membrane exocytosis and lipid modifications (Wodarz \& Nathke, 2007;

\footnotetext{
${ }^{1}$ Department of Medicine, University of California San Diego, La Jolla, CA, USA ${ }^{2}$ Department of Cellular and Molecular Medicine, University of California San Diego, La Jolla, CA, USA ${ }^{3}$ Moores Cancer Center at UC San Diego Health, La Jolla, CA, USA ${ }^{4}$ Veterans Affairs Medical Center, La Jolla, CA, USA ${ }^{5}$ Department of Pathology, University of California San Diego, La Jolla, CA, USA ${ }^{6}$ Institut National de la Santé et de la Recherche Médicale (French Institute of Health and Medical Research) (INSERM) U1016, Institut Cochin, Paris, France ${ }^{7}$ Centre National de la Recherche Scientifique (National Center for Scientific Research) (CNRS) United for Medical Research (UMR) 8104, Paris, France ${ }^{8}$ Université Paris Descartes, Sorbonne Paris Cité, Paris, France ${ }^{9}$ Department of Pediatrics, University of California San Diego, La Jolla, CA, USA ${ }^{10}$ Department of Computer Science and Engineering, Jacob's School of Engineering, University of California San Diego, La Jolla, CA, USA ${ }^{11}$ Microbiology and Immunology Department, Assiut University, Asyut, Egypt
} 
St Johnston \& Ahringer, 2010). Augmentation of epithelial polarity requires an additional signaling component which is triggered exclusively in response to stress. Three studies (Zhang et al, 2006; Lee et al, 2007; Zheng \& Cantley, 2007) reported a role of AMP-activated protein kinase (AMPK) in the maintenance of epithelial cell polarity and barrier functions in the context of stress; who or what was its effector at the cell-cell junctions remained unknown. A decade later, Aznar et al (2016) demonstrated that GIV ( $\mathrm{G}-\alpha$ interacting vesicle-associated protein, also known as Girdin), a multimodular polarity scaffold protein is a substrate of AMPK and defined the molecular mechanisms by which the AMPKGIV signaling axis protects the epithelium by stabilizing tight junctions (TJS) and preserving cell polarity when challenged with energetic stress. Using MDCK cells as a model of polarized mammalian cells, Aznar et al (2016) showed that energetic stress triggers localized activation of AMPK at the tricellular TJs which mark the most vulnerable cell-cell contacts in sheets of polarized cells. A significant part of the junction-stabilizing effects of AMPK agonists such as 5-Aminoimidazole-4-carboxamide ribonucleotide (AICAR) and metformin during energetic stress (Zhang et al, 2006; Zheng \& Cantley, 2007) appeared to be mediated by AMPK via its downstream effector, pS245-GIV (Aznar et al, 2016). Based on these findings, the AMPK $\rightarrow$ pS245 GIV signaling axis was named the "Stress Polarity Signaling (SPS)-Pathway." It was shown that the SPS-pathway inhibits oncogenic transformation and anchorage-independent growth of cancer cells; disruption of this pathway via mutations that make GIV non-phosphorylatable helps tumor cells escape such inhibition and gain proliferative advantage. These findings led to the conclusion that the SPS-pathway integrates stress-sensing and augmention of cell polarity pathways to serve as a deterrent to oncogenesis.

Despite these insights, the pathophysiologic relevance of such integration illustrated exclusively in cell lines, remained unclear. Here, we used a near-physiologic model system (co-cultures of microbes with mouse and human organoids) to assess the importance of this pathway in the gut lining. Our studies reveal the physiologic importance of the SPS-pathway in the maintenance of the gut barrier function and consequences when it is dysregulated. Findings also reveal the therapeutic potential of AMPK agonists such as metformin in strengthening the gut barrier. Over the years, the beneficial (protective) effect of multiple nutritional components, dietary supplements, and pharmacologic agents, including the widely prescribed AMPK-activator, metformin, on intestinal permeability in health and disease has been investigated; all studies converge on AMPK activation as a common pre-requisite for rendering such protection (reviewed in (Ghosh (2017)). Taken together with these prior works, our findings provide an impetus for harnessing the diagnostic and therapeutic potential of the SPSpathway in the setting of a variety of diseases that are associated with increased intestinal permeability such as inflammaging and cancer.

\section{Results and Discussion}

\section{The SPS-pathway is active in the colon epithelium and requires the catalytic activity of AMPK}

First, we asked if the SPS-pathway is active in the epithelial lining of the human colon. To this end, we performed immunohistochemistry (IHC) using the previously validated (Aznar et al, 2016) anti-pS245-GIV antibody on formalin-fixed paraffin-embedded (FFPE) colon tissues obtained during routine colonoscopy for colon cancer screening (Fig 1A). Although all tissues were histologically confirmed as "normal," some of them were from patients with type II diabetes on chronic therapy with metformin at doses ranging from 500 to $1,500 \mathrm{mg} / \mathrm{d}$. We preferred the use of pS245-GIV as a "surrogate marker" of the SPSpathway (as opposed to other global anti-phospho-AMPK antibodies) because pS245-GIV was previously determined (Aznar et al, 2016) to be the TJ-localized substrate of AMPK activity which is both necessary and sufficient for protecting TJs from stress-induced collapse. It offers a way to specifically monitor AMPK's TJ-stabilizing effect as opposed to its general effect on cellular bioenergetics. In fact, a side-by-side comparison of the two targets showed that although pS245GIV remained associated with TJs in a sustained manner, pAMPK is activated only transiently at tricellular contact site and then shows a cytosolic staining pattern around disrupted TJs (Aznar et al, 2016). We found that although the intensity of staining varies among patients, a larger proportion of patients on metformin displayed positive staining and at a stronger intensity compared with the cohort of patients, not on metformin (Figs $1 \mathrm{~A}$ and S1). These findings indicate that the SPSpathway, as determined by pS245-GIV as a surrogate marker, is active in the human colon epithelium and that although its degree of activation varies considerably in the normal colon, it appears to be consistently enhanced in patients exposed to the widely prescribed AMPK-agonist metformin.

To translate the physiological implications of the observations made using polarized MDCK monolayers (Aznar et al, 2016), we used an ex vivo near-physiologic model system called the "gut-in-adish" (see the legend and Fig 1B). In this model, crypt-derived stem cells isolated from human or mouse colon (see the Materials and Methods section) were used to generate organoids and later differentiated into polarized enteriod-derived monolayers (EDMs). These EDMs have been validated as model systems that closely resemble the physiologic gut lining in which all cell types (enterocytes, goblet, Paneth enteroendocrine, and tuft cells) are proportionately represented (Sato et al, 2009; Miyoshi \& Stappenbeck, 2013; Foulke-Abel et al, 2014; Noel et al, 2017; Yu et al, 2017). We used this "gut-in-a-dish" model to rigorously interrogate the impact of various stressors on the integrity of the gut barrier and cellular processes, using readouts illustrated in Fig 1B. Using pS245-GIV as a marker, we confirmed that the SPSpathway is active in the EDMs, colocalized with the TJ protein, occludin, as previously shown in MDCK cells; (Aznar et al, 2016). When we treated the EDMs with metformin, the pathway was significantly enhanced-whereas immunofluorescence studies (Figs 1C-Left and S2) showed a approximately fivefold to sixfold increase in the intensity of occludin staining, immunoblots (Fig 1C-Right) showed a peak increase in pS245 GIV and PAMPK up to approximately twofold to threefold at $4 \mathrm{~h}$.

Next, we asked if activation of the SPS-pathway in the gut epithelium requires the catalytic activity of AMPK. We assessed the levels of pS245-GIV in enteroids using two parallel approaches: 1) We generated enteroids from AMPK $\alpha 1 / 2$-Villin-Cre mice (generated by Benoit Viollet, Institut National de la Santé et de la Recherche Médicale (French Institute of Health and Medical Research) [INSERM]; manuscript in preparation) which lack both the $\alpha$-isoforms that encode the catalytic subunits of the heterotrimeric kinase. 2) We used a well-validated direct agonist of AMPK, A-769662 (Cool et al, 2006), for 
A Immunohistochemistry (IHC) Healthy Controls $\mathrm{p}<0.0001$

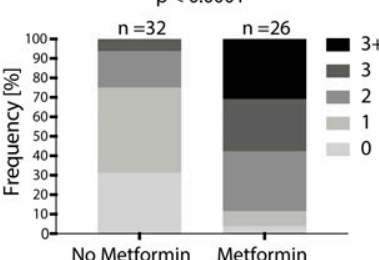

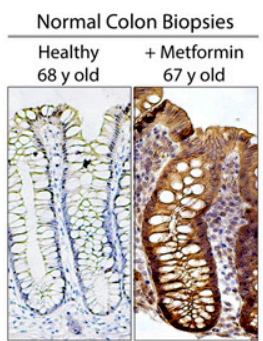

B

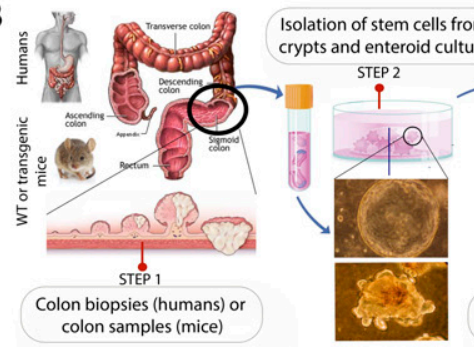

Readouts of barrier integrity TEER, confocal microscopy of $T J$ markers 2. Cytokine production: 9 PCR, EUSA, 3. Gene regulatory changes: $\mathrm{QPCR}$

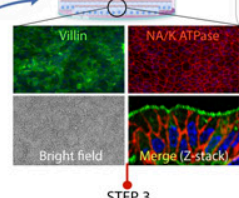

TEP 3

ion into Enteroidderived Monolayers (EDMs)

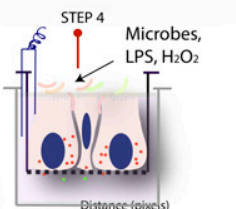

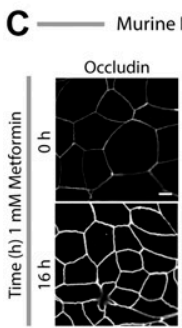
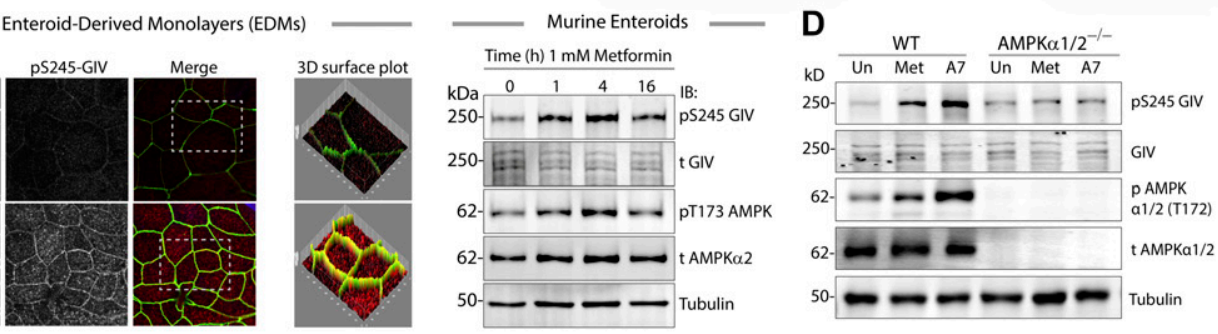

E
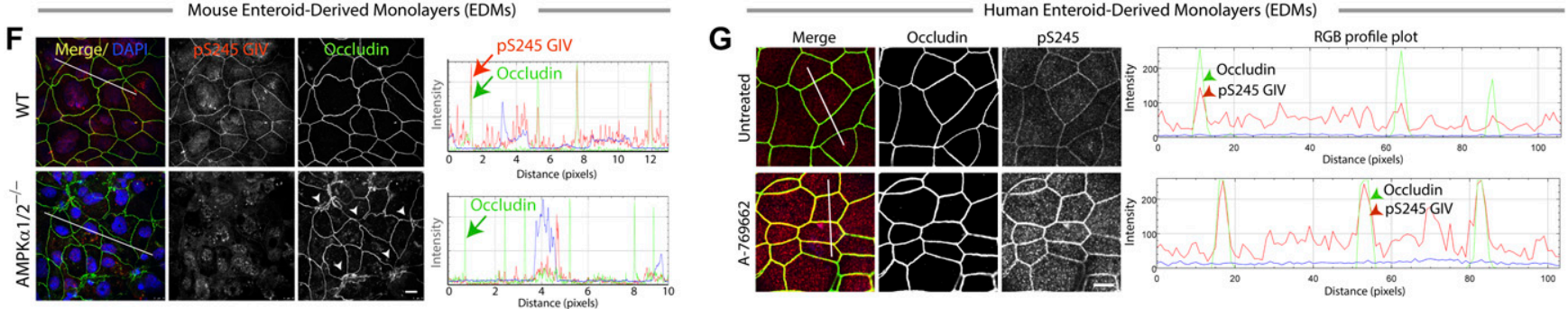

Figure 1. The stress polarity signaling (SPS) pathway is active in the gut lining, and its pharmacologic augmentation requires the catalytic activity of AMP-kinase. (A) Left: The SPS-pathway was evaluated in normal adult colon by immunohistochemistry on FFPE colonic biopsies using anti-pS245-GIV, and the staining intensity in the epithelium was scored (see Fig S1). Bar graph displays the proportion of patients in each group with varying intensities of staining. Two-sided Fisher's exact test was used to calculate significance. Right: Representative images are presented from healthy adult without metformin (lowest "0" staining) and with metformin (highest " $>3+$ " staining) within the cohort. (B) Schematic showing the key steps involved during the development of the stem cell-based organoid model, "gut-in-a-dish." Fresh biopsies obtained from the colons of mice and humans (STEP 1) are used as source of stem cells to grow organoids (STEP 2). Organoids are differentiated into polarized enteroid-derived monolayers (EDMS; STEP 3) for co-culture studies with microbes and microbial products (exposed apical surface) to mimic the gut lumen in physiology and enable the assessment of barrier integrity (STEP 4). (C) Left: Murine EDMs treated with metformin for 0, 1, 4, and $16 \mathrm{~h}$ were fixed; stained with anti-pS245-GIV (red; a surrogate measure of the SPS-pathway), occludin (green; a bonafide tight junction [TJ] marker), and nucleus (DAPI); and analyzed by confocal microscopy. Images (left) display the findings at 0 and $16 \mathrm{~h}$; see Fig S2 for all intermediate time points and for quantification of pixel intensity of occludin staining at TJs. Boxed areas were analyzed by 3D surface plot (Image J). Scale bars $=10 \mu \mathrm{m}$. Right: Immunoblots (right) on whole cell lysates of murine enteroids treated for 0, 1, 4, and $16 \mathrm{~h}$ incubated with anti-phospho(p) and anti-total(t) GIV and antiAMPK proteins, and $\alpha$-tubulin (as loading control) confirm activation of the SPS pathway in murine enteroids. Images and immunoblots presented are representative of $\sim 3-5$ independent experiments. (D) Enteroids isolated from WT or AMPKo1/2-1- mice were treated or not (“Un") with the indicated pharmacologic agonists of AMPK (1 mM Met, metformin; $100 \mu \mathrm{M}$ A7, A769662) for $4 \mathrm{~h}$ before lysis. Equal aliquots of whole cell lysates were analyzed for activation of AMPK and the SPS-pathway by immunoblotting. (E) Bar graphs display the change in trans-epithelial electrical resistance (TEER) across EDMs prepared from WT and AMPKo1/2-1-mice. Findings were analyzed by one-way ANOVA followed by multiple comparison test. Error bars represent mean $\pm \mathrm{SEM}, \mathrm{n}=3$; ${ }^{* *} \mathrm{P}<0.01$. (B, F) Murine EDMs derived from WT or AMPKa $1 / 2^{-1-}$ mouse colons were prepared as described in (B), fixed, and analyzed for the integrity of the epithelial tight junctions and the SPS-pathway using anti-occludin and anti-pS245GIV, respectively, and imaged by confocal microscopy. Left: Images displayed are representative of three independent experiments. Arrowheads point to "burst" pattern of occludin staining at the disrupted tricellular TJs. Scale bars $=10 \mu \mathrm{m}$. Right: RGB profile plot (right) indicate the co-localization of pS245-GIV and occludin as assessed using an Imagej plug-ins. See also Fig S3 for the effect of AMPK agonist on EDM. (G) Human EDMs were either untreated or treated with A-769662 (100 $\mu \mathrm{M})$ for $16 \mathrm{~h}$; fixed; stained with anti-pS245-GIV (red; a surrogate measure of the SPS-pathway), occludin (green; a bonafide TJ marker), and nucleus (DAPI); and analyzed by confocal microscopy. Left: Images displayed are representative of three independent experiments. Scale bars $=10 \mu \mathrm{m}$. Right: RGB profile plot (right) indicated the co-localization of pS245-GIV and occludin as assessed using an ImageJ plug-ins.

which the structural basis for activation of the heterotrimer is known (Xiao et al, 2013). We found that neither metformin nor A7 could induce pS245-GIV in enteroids devoid of AMPKa1/2 (Fig 1D). Compared with EDMs from WT mice, EDMs derived from AMPK $\alpha 1 / 2-$ Villin-Cre mice showed impaired barrier integrity and higher paracellular permeability, as reflected by low trans-epithelial electrical resistance (TEER; Fig 1E), absence of pS245-GIV staining at the TJs, and aberrant TJ morphology detected using occludin a bona fide TJ protein ("burst" appearance of tricellular TJs; see arrowheads, Fig 1F). Although pS245GIV was virtually absent at TJS, some nuclear speckles were observed in AMPK $\alpha 1 / 2$-Villin-Cre EDMS, the significance of which remains unknown. Furthermore, when we treated the WT or AMPKa1/2-Villin-Cre EDMs with A-769662, the SPSpathway was enhanced in the WT EDMS, as determined by increased intensities of both occludin and pS245GIV, but the pathway could not be rescued in the AMPKa1/2-Villin-Cre EDMs (Fig S3). The AMPK agonists also activated the SPS-pathway in human EDMs (Fig 1G). Together, these results using a combination of AMPK-depleted EDMs and highly specific AMPK agonist demonstrate that the SPS-pathway, as determined by the abundance of pS245-GIV in the gut epithelium, 
requires the metabolic kinase AMPK, and its absence compromises the integrity of the epithelial barrier.

The SPS-pathway protects the gut barrier against diverse stressors such as microbes and microbial products

Next, we used the "gut-in-a-dish" model to study the role of the SPS-pathway in EDMs under stress. Our choice of stressors included those that are physiologically encountered within the gut lumen, for example, a) live commensal microbes (Escherichia coli; Fig $2 \mathrm{~A}-\mathrm{C}$ ), b) microbial outer membrane components (LPS; Fig 2D and $\mathrm{E}$ ) and $\mathrm{H}_{2} \mathrm{O}_{2}$ (Fig $2 \mathrm{D}$ and $\mathrm{F}$ ); the latter induces reactive oxygen species in ways that mimic the host response to dysbiosis or in response to injury or inflammation. EDMs were either treated or not with metformin followed by exposure to each stressor. EDMs were assessed for barrier integrity by analyzing the same sample by two
A

A

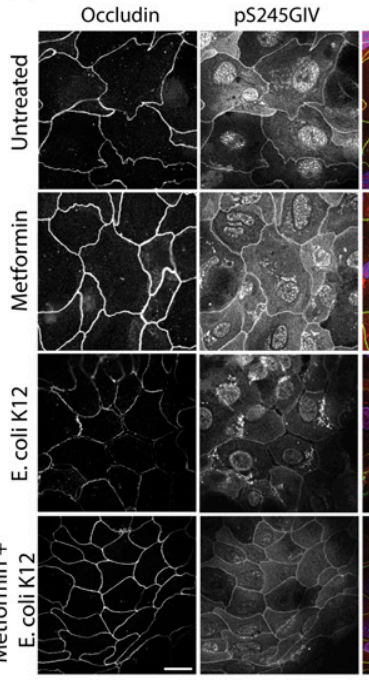

Mouse Enteroid-Derived Monolayers (EDMs)
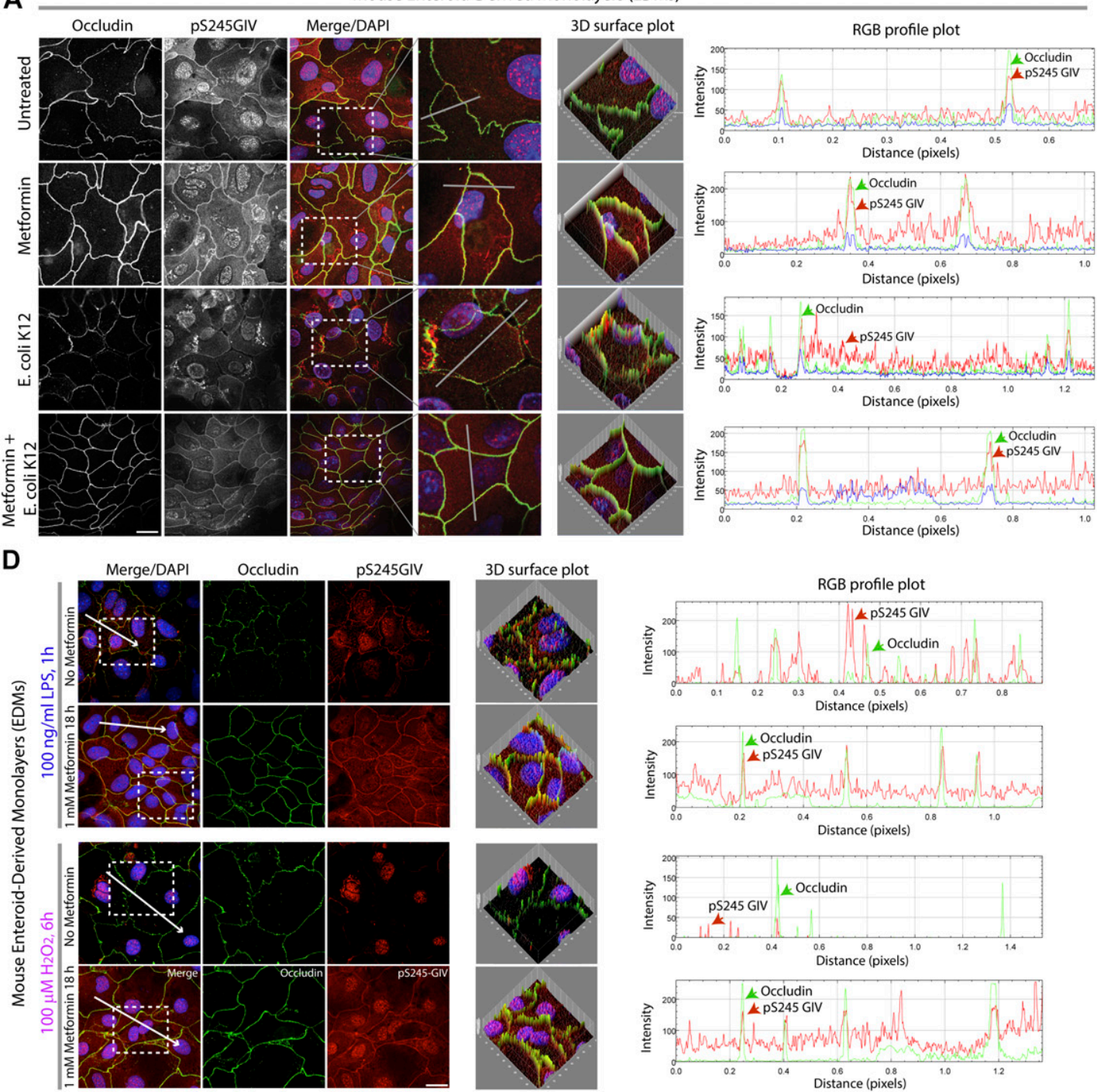

$\mathbf{F}$

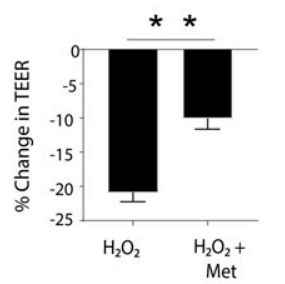

Figure 2. Pharmacologic augmentation of the stress polarity signaling (SPS) pathway protects the gut barrier against diverse stressors such as microbes and microbial products.

(A) Mouse enteroid-derived monolayers (EDMs) were infected with E. coli K12 with or without prior exposure to metformin (1 mM for 16 h); fixed; stained with anti-pS245GIV (red; a surrogate measure of the SPS-pathway), occludin (green; a bona fide tight junction [TJ] marker), and nucleus (DAPI); and analyzed by confocal microscopy. Left Images displayed are representative of three independent experiments. (A) Co-localization of pS245-GIV and occludin was assessed using ImageJ plug-ins: RGB profile plot (A-middle) and 3D surface plots (A-right). Scale bars $=10 \mu \mathrm{m}$. (A, B) Bar graphs display the \% of TJs that appeared broken and/or splitting ("burst"-appearing) on the $y$-axis encountered in 8-10 randomly chosen fields from three independent experiments in (A). Findings were analyzed by one-way ANOVA followed by multiple comparisons test. Error bars represent mean $\pm \mathrm{SEM}, \mathrm{n}=3$; ${ }^{*} \mathrm{P}<0.01$. (A, C) Bar graphs display the change in TEER across the EDMs in (A). Findings were analyzed by oneway ANOVA followed by multiple comparison test. Error bars represent mean $\pm \mathrm{SEM} ; \mathrm{n}=3$; ${ }^{*} \mathrm{P}<0.01$. (D) Mouse EDMs pretreated (or not) with metformin ( $1 \mathrm{mM}$ for $16 \mathrm{~h}$ ) were exposed to LPS or $\mathrm{H}_{2} \mathrm{O}_{2}$, as indicated; fixed; stained with anti-pS245-GIV (red; a surrogate measure of the SPS-pathway), occludin (green; a bonafide TJ marker), and nucleus (DAPI); and analyzed by confocal microscopy. Left: Images displayed are representative of three independent experiments. Scale bars = $10 \mu \mathrm{m}$. Right: Colocalization of pS245-GIV and occludin was assessed using ImageJ plug-ins, RGB profile plot. (D, E, F) Bar graphs display the change in TEER of the EDMs after LPS (E) or $\mathrm{H}_{2} \mathrm{O}_{2}(\mathrm{~F})$ treatment shown in (D). Findings were analyzed by one-way ANOVA followed by multiple comparisons test. Error bars represent mean $\pm \mathrm{SEM}$; $\mathrm{n}=3$; ${ }^{\star *} \mathrm{P}<0.01$. 
assays: a) periodic measurements of TEER and b) immunofluorescence with pS245-GIV and occludin to assess the SPS-pathway and TJ morphology, respectively. We continued to monitor occludin as a readout of TJ morphology because this integral membrane protein allows us to not just visualize but also quantify the degree of TJ disruption ("burst" tricellular TJs where three or more cells come in contact [Furuse et al, 2014]); the tricellular TJs appear to be the regions where earliest evidence of disruption can be visualized/ assessed first. Also, prior work (Aznar et al, 2016) showed that under stress, active AMPK was detected transiently at the TJs of tricellular contact, as determined by colocalization of phospho-AMPK with occludin. We found that all stressors induced barrier disruption in untreated EDMs, as determined by occludin staining (Fig 2A, B, and D) and by the observed drops in TEER (Fig 2B, C, E, and F). Pretreatment of EDMs with metformin showed SPS-pathway activation (pS245), maintenance of TJ architecture (occludin), and preservation of barrier integrity (TEER). We conclude that treatment with metformin resists barrier collapse in all stress-inducing conditions tested, implicating the barrier-protective role of the SPS-pathway in the gut epithelium.

\section{The SPS-pathway is suppressed in the aged gut; its loss triggers inflammation}

Among the various organ systems that decline during aging, dysfunction of the intestinal barrier has been correlated with increasing age in a variety of species. For example, dysfunction of the intestinal barrier predicts impending death in individual flies regardless of their chronological age (Rera et al, 2012). Much like humans, dysregulation of barrier in these flies shows an age-related increase in immunity-related gene expression (e.g., IL-6) (Rera et al, 2012). Evidence also shows that intestinal barrier dysfunction during aging is conserved in worms (Caenorhabditis elegans), fish (Danio rerio) (Tricoire \& Rera, 2015; Dambroise et al, 2016), and mammals (rats [Katz et al, 1987] and baboons [Tran and Greenwood-Van Meerveld, 2013]), thus raising the possibility that it may also be the case in humans. To investigate this, we first characterized human enteroids derived from term male fetuses and "normal" colon biopsies from elderly male subjects (Fig 3A) for transcript expression levels of SIRTs 1 and 6, two key aging/longevity related families of proteins with either monoADP-ribosyltransferase or deacylase activity, that is, sirtuins because previous lifespan studies carried out using yeast, worms, and flies as model organisms have demonstrated that sirtuins are evolutionarily conserved mediators of longevity (Kaeberlein et al, 1999; Tissenbaum \& Guarente, 2001; Rogina \& Helfand, 2004). Both SIRTs have been shown to regulate inflammation in the gut (Akimova et al, 2014; Lo Sasso et al, 2014; Wellman et al, 2017; Zhang et al, 2018). We found that compared with fetal EDMs, aged EDMs expressed less SIRT1 and 6 (Fig 3B-Left, Middle), consistent with the previous observations of their decline in the aged gut (Liu et al, 2017a; Igarashi et al, 2019). Because age-associated changes in gut microbiome composition is correlated with increases in the pro-inflammatory marker serum monocyte chemoattractant protein (MCP-1) (Conley et al, 2016), which in turn has been implicated in aging-related macrophage dysfunction (Thevaranjan et al, 2017), we analyzed this cytokine in the aged EDMs. We found that MCP-1 transcript levels were increased in aged EDMS relative to fetal EMDs (Fig 3B-Right).
Increased intestinal leakiness in the aged gut has been documented (Valentini et al, 2014). Therefore, next, we analyzed the barrier integrity of and the status of the SPS-pathway in the aged EDM. We found by confocal microscopy that TJs were more frequently disrupted in the aged compared with fetal EDMs (Fig 3C; occludin signal) and that the SPS-pathway is suppressed (Fig 3C; pS245-GIV signal was virtually undetectable). Treatment of the aged EDMS with metformin restored the SPS-pathway, repaired the "burst" TJs (visualized by occludin staining) (Fig 3C), and increased TEER (Fig 3D). Pharmacologic activation of the SPS-pathway either with metformin or the AMPK-specific activator, A-769662 (Fig 3C-H), reduced the leaky TJ-protein, claudin-2 (CLDN2) (Fig 3E), and suppressed proinflammatory cytokines (IL8, IL6, and MCP1) (Fig 3F-H). These findings indicate that pharmacologic activation of AMPK and restoration of the SPS-pathway is sufficient to reduce proinflammatory cytokines that are seen in the aged EDMs. Finally, we confirmed that the SPS-pathway is indeed suppressed in the aged gut lining, as determined by IHC on FFPE colon tissues obtained during routine colonoscopy from patients representative of various age groups (Fig 3I). Taken together, we conclude that the SPS-pathway is suppressed in the aged gut epithelium, and such suppression is permissive to a pro-inflammatory program.

\section{The SPS-pathway is suppressed during colorectal cancer (CRC) initiation and progression}

Previously, Aznar et al (2016) indicated the tumor-suppressive property of the SPS-pathway. Here, we investigated the activation status of the tumor-suppressive SPS-pathway during CRC initiation and progression. Because TJ-localized pS245-GIV serves as a functional molecular marker that reflects the activation state of the SPS-pathway, we carried out IHC with anti-pS245-GIV on FFPE colon tissues from various stages of normal-to-cancer progression in the colon (Fig 3J). Phospho-S245-GIV was undetectable in almost all advanced adenomas and all CRCs analyzed (Fig 3K and L), indicating that the SPS-pathway is lost or silenced during CRC progression. This finding is consistent with our previous findings (Aznar et al, 2016) demonstrating the tumor-suppressive property of the SPS-pathway in 3D-cultured DLD1 CRC cell lines. It is important to note that the pattern of pS245GIV staining is strikingly different from the levels of total GIV during CRC initiation and progression; we have previously shown that GIV mRNA and protein generally decreases first during polyp formation and progression and subsequently increases during CRC progression to metastasis (Ghosh et al, 2010). We and others have shown that increased levels of GIV in established CRCs correlate with aggressive features (GarciaMarcos et al, 2011b; Jun et al, 2013; Aznar et al, 2016; Barbazan et al, 2016; Ghosh et al, 2016; Lu et al, 2018), for example, shorter metastasis-free survival, chemoresistance, and stemness, largely attributed to its ability to scaffold multi-receptor signaling cascades and enhance them via $G$ protein intermediates to trigger epithelial mesenchymal transition (reviewed in Ghosh (2015)). Taken together, the loss of pS245 GIV early during polyp to CRC progression underscores how GIV may serve as a tumor suppressor when localized to TJs in the polarized normal epithelium before it assumes its role as an enhancer of epithelial-mesenchymal transition and stemness in established CRCS. 
A

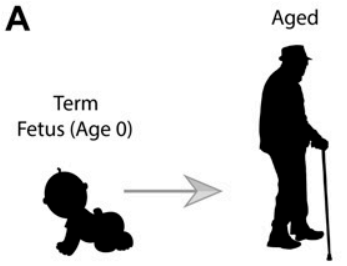

C

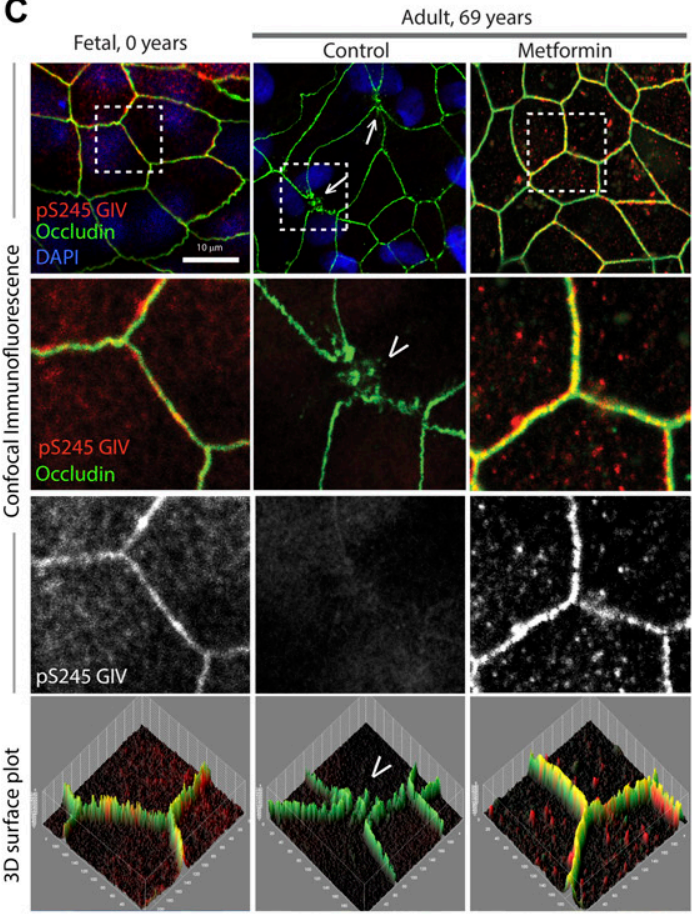

G
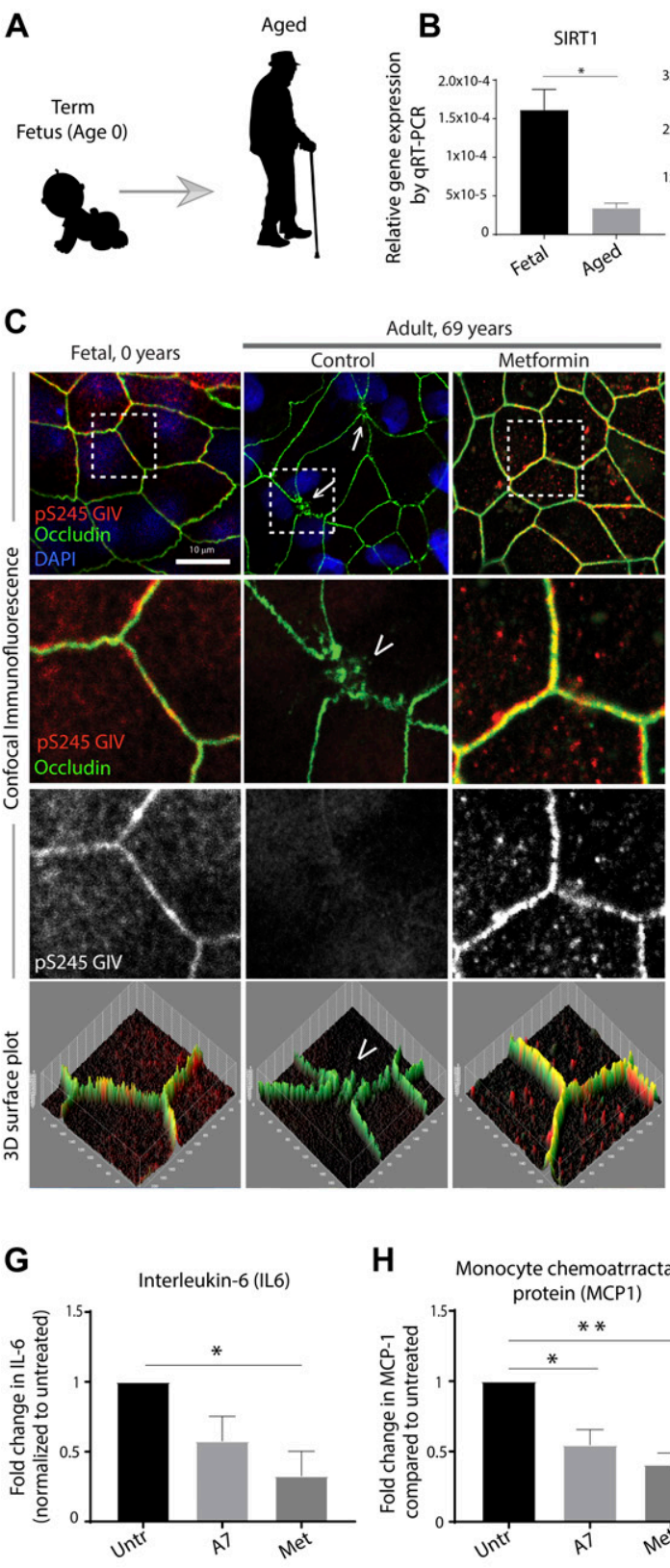
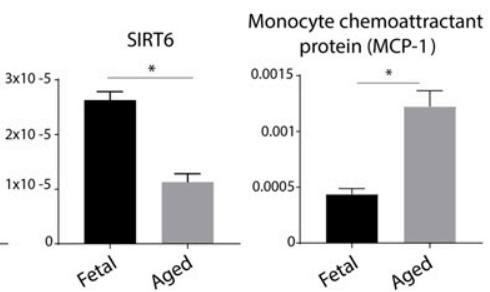

D

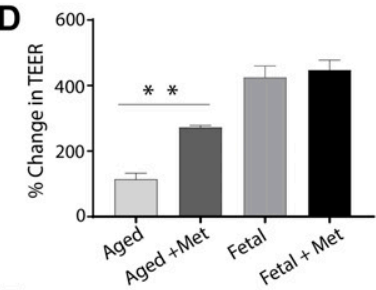

E
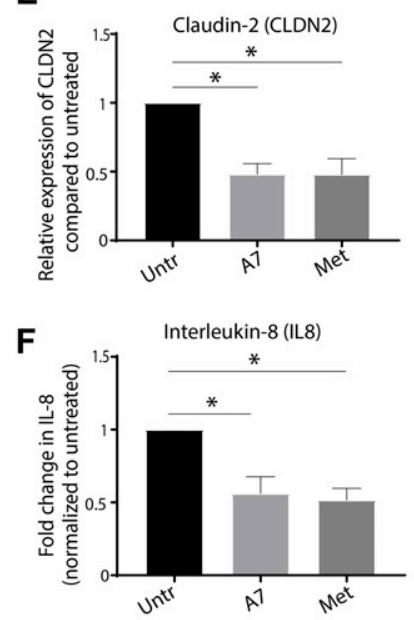

H Monocyte chemoatractant

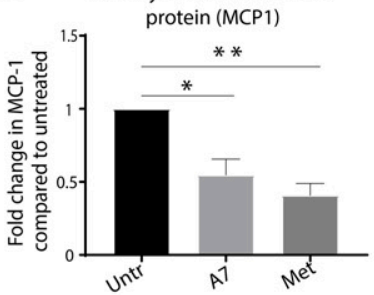

I IHC: pS245 GIV [Healthy Controls] $\mathrm{p}=0.0311$

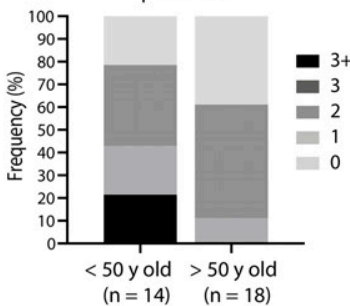

J Normal Early Intermed Advanced Cancers
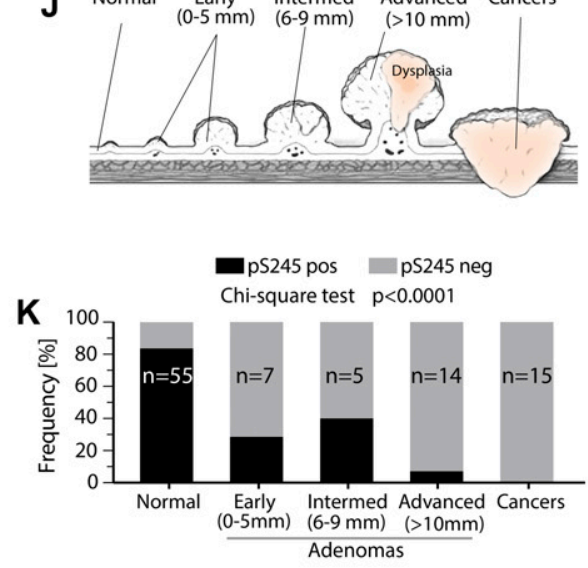

$\mathbf{L}$
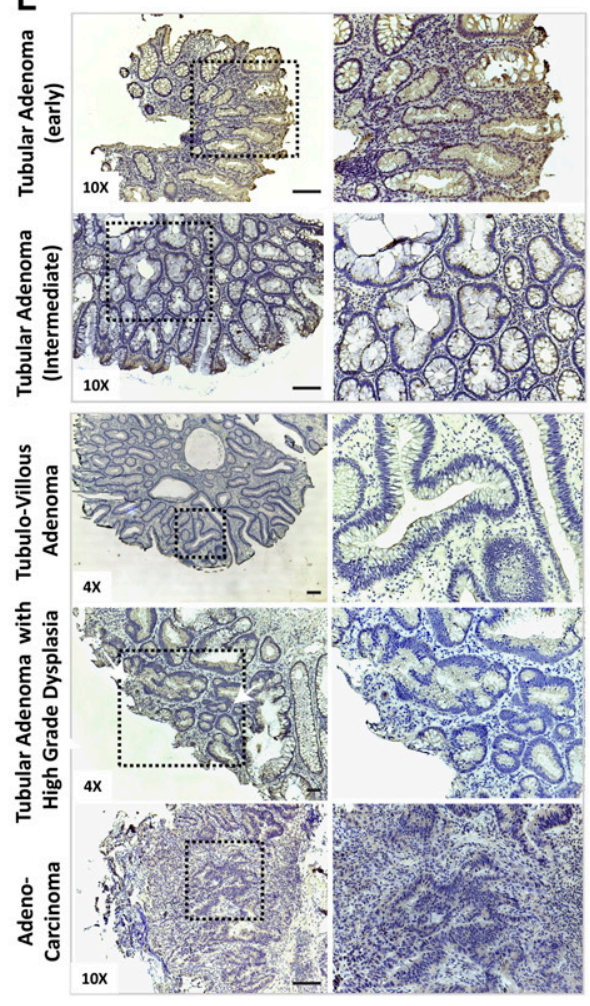

Figure 3. Stress polarity signaling pathway is impaired in the aging gut and during the initiation and progression of colorectal cancers.

(A, B, C, D, E, F, G, H) Schematic showing the profile of human enteroid-derived monolayers (EDMs) used in panels (B, C, D, E, F, G, H). Term fetus is indicated as age 0 yr. (B) Fetal ( $0 \mathrm{yr} ; \mathrm{n}=3$ subjects) and aged ( $\sim 50-70$-yr old; $\mathrm{n}=3$ subjects) human EDMs were analyzed for aging molecules, Sirtuins (SIRT) 1 and 6 , and MCP-1 by qRT-PCR. Data displayed as mean \pm SEM from three independent experiments. Findings were analyzed by one-way ANOVA followed by multiple comparisons test. ${ }^{*}=P<0.05$. $(\mathbf{B}, \mathbf{C})$ EDMs in (B) were pretreated or not with metformin ( $1 \mathrm{mM}$ for $16 \mathrm{~h}$ ); fixed; stained with anti-pS245-GIV (red; a surrogate measure of the stress polarity signaling-pathway), occludin (green; a bonafide tight junction marker), and nucleus (DAPI); and analyzed by confocal microscopy. Above: Images displayed are representative of three independent experiments. Scale bars $=10 \mu \mathrm{m}$. Co-localization of pS245-GIV and occludin was assessed using Image plug-ins and below, 3D surface plots. (C, D) Bar graphs display the change in TEER of the EDMs in (C). Findings were analyzed by two-way ANOVA followed by multiple comparisons test. Error bars represent mean \pm SEM; $n=3$; ${ }^{* *} \mathrm{P}<0.01$. (E, F, G, H) The aged (69-yr-old) human EDMs were treated or not (“Untr") with metformin (1 mM) or A7 (A769662; $100 \mu \mathrm{M}$ ) for $16 \mathrm{~h}$ before analyzing them for the levels of expression of CLDN2 (claudin-2), IL-8, IL-6, and MCP-1 by qRT-PCR. Data displayed as mean \pm SEM from three independent experiments normalized to untreated control. Findings were analyzed by two-way ANOVA followed by multiple comparisons test. ${ }^{*}=P<0.05$; ${ }^{* *}=P<0.01$. (I) FFPE human colon tissues representing young ( $<50$ $y r ; n=14$ ) or aged ( $>50 \mathrm{yr} ; \mathrm{n}=18$ ) were analyzed for pS245-GIV by immunohistochemistry, and the staining intensity in the epithelium was scored (see Fig 1 Supplement 2). Bar graph displays the proportion of patients in each group with varying intensities of staining. Two-sided Fisher's exact test was used to calculate significance. (J, K, L) FFPE human colon tissues representing normal, adenomas, and carcinomas (schematic, J) were analyzed for pS245-GIV by immunohistochemistry. Bar graph ( $K$ ) displays the proportion of samples in each category that stained positive. Two-sided Fisher's exact test was used to calculate significance. (L) Images representative of each category are displayed (L). Panels on the right are magnified boxed areas of the images on the left. Scale bar $=100 \mu \mathrm{m}$. 


\section{Conclusion}

The importance of the gut barrier has gained so much traction in the past decade that it has ushered in the dawn of "barriology" (Tsukita et al, 2008). The current thinking in the field of "barriology" is that chronic systemic endotoxemia, due to a compromised gut barrier in the setting of stress, impacts multiple diseases. Microbial dysbiosis is known to trigger major cellular programs, for example, loosening of cell-cell junctions, loss of cell polarity, and chronic inflammation, all of which coordinately fuel the progression of these chronic diseases. This work reveals the physiologic importance and therapeutic potential of the SPS-pathway to resist and reset gut microbe triggered programs by fortifying epithelial TJs (see Summary and working model; Fig 4). Using the combined synergy of patient-derived tissues and mouse and human organoid-based models, we showed that the SPS-pathway serves as a protective host response that is compromised in the aged gut and early during the initiation of colon cancers.

This work highlights the SPS-pathway as an "actionable pathway" in the gut barrier. The abundance of TJ-localized pS245-GIV serves as a reliable marker of the SPS-pathway and a surrogate measure of the integrity of the gut barrier. These are important findings because despite decades of research showing the importance of the gut barrier in health and disease and discovery of plausible targets (Cunningham \& Turner, 2012), our ability to detect and fix a leaky gut barrier is not currently a practical option in clinical practice. By demonstrating direct and specific AMPK-agonists as effective augmentors of gut barrier integrity and by validating pS245-GIV as a reliable molecular marker to track such pharmacologic augmentation, this work translates the therapeutic and diagnostic potential of the fundamental discoveries made by Aznar et al (2016).

With regard to the impact of our findings in human diseases, because chronic low-grade inflammatory status in the aging gut has led to coining of the term "inflammaging" (Giunta, 2006; Franceschi, 2007; Franceschi et al, 2007; Goto, 2008; Minciullo et al, 2016; Manabe, 2017), our findings suggest that activation of the SPS-pathway may serve as a strategy to combat this entity. Because the SPS-pathway was initially characterized as a tumor-suppressive pathway, and we have now shown that this pathway is silenced early during CRC initiation, these findings suggest that activation of the SPS-pathway may serve as a strategy to prevent polyp-to-cancer progression in the colon. Our findings also raise the possibility that the SPSpathway may affect a variety of non-oncologic diseases that are associated with increased intestinal permeability (reviewed in Bischoff et al (2014)) such as chronic gastrointestinal inflammation, Alzheimer's, Parkinson's, multiple sclerosis, autism, chronic heart failure, and obesity and metabolic diseases. All these diseases are characterized by systemic inflammation due to chronic endotoxemia that might be triggered by the translocation of endotoxins from the gut lumen into the host circulation.

By demonstrating the therapeutic potential of metformin as an activator of the SPS-pathway, our findings revisit the mechanism of action of this first-line treatment for type II diabetes. Although metformin (Glucophage) is now the most widely prescribed type II diabetes drug in the world for its ability to reduce blood glucose by activating the LKB1-AMPK pathway (Shaw et al, 2005) and inhibiting hepatic gluconeogenesis (reviewed in Shackelford and Shaw (2009)), it is also known to exert other effects in an AMPKdependent manner: (i) it stabilizes cell-cell junctions and protects barrier functions of both epithelial and endothelial monolayers in the setting of a variety of pathologic stressors and (ii) it suppresses the growth of a variety of tumor cells and embryonic stem cells in culture and tumor xenografts in mice (reviewed in Shackelford and Shaw (2009)). Numerous studies using the AMPK-activator, metformin, squarely implicate the AMPK-dependent stress polarity pathway as a major therapeutic target in these metabolic disorders (Everard et al, 2013; Buse et al, 2016; Zhou et al, 2016). metformin administration enhances gut barrier integrity, attenuates endotoxemia, and enhances insulin signaling in high-fat fed mice, which accounts for the beneficial effects of metformin on glucose metabolism, enhanced metabolic insulin response, and reduced oxidative stress in liver and muscle of the mice (Zhou et al, 2016). It is possible that the observed anti-ageing properties of metformin (via multiple widely pleiotropic effects reviewed in Barzilai et al (2016)), as in the case of obesity and diabetes, may begin by preserving the gut barrier function, thereby reducing age-related inflammation and metabolic derangements. If so, metformin is expected to act via the AMPK-GIV SPS-pathway to resist aging-related increase in gut permeability. Ongoing clinical

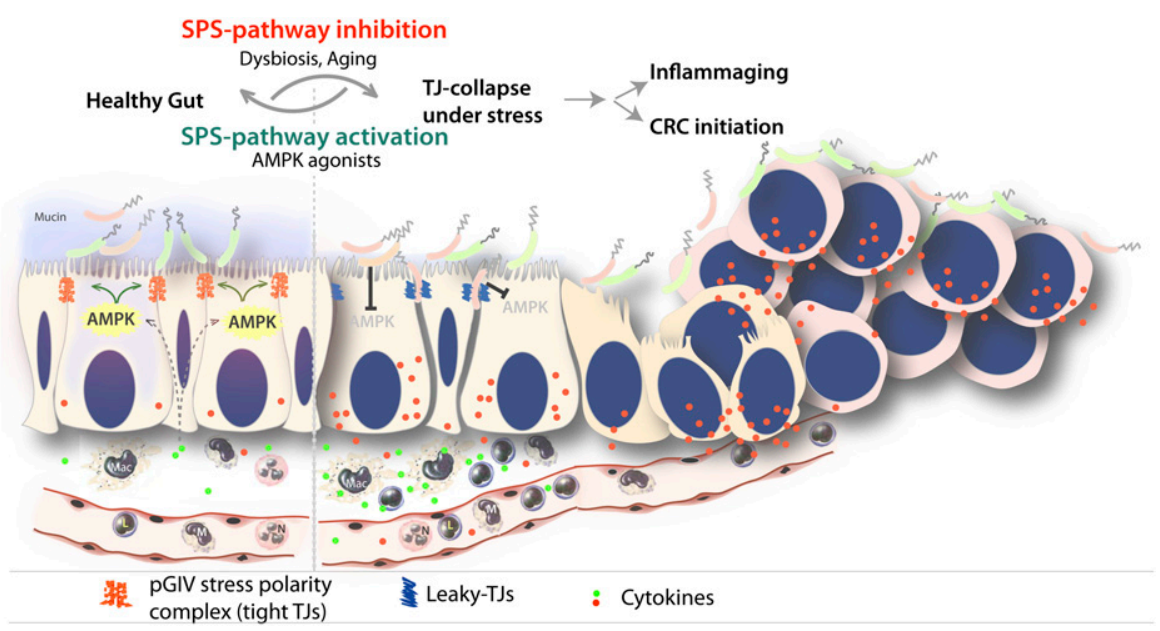

Figure 4. Summary of findings and working model. Summary and proposed working model for the role of the stress polarity signaling (SPS) pathway in the gut barrier. Genetic, epigenetic, or dysbiosis-induced inhibition of the SPS-pathway leading to stressinduced tight junction-collapse and loss of cell polarity. Loss of the SPS-pathway (in aging and during cancer initiation) is accompanied by collapse of epithelial tight junctions and loss of cell polarity, which is permissive to a gene expression signature that promotes leakiness of the gut barrier (high claudin2) and inflammation (MCP1, IL8). Pharmacologic augmentation of the SPS-pathway with AMPK agonists may resist and reverse the above. 
trials approved by the Food and Drug Administration (such as Targeting Ageing with Metformin; TAME) are likely to provide the best opportunity to investigate these possibilities. In fact, one clinical trial already hints at that. Using a delayed release formulation of metformin (metformin DR, which is designed to target the lower bowel and limit absorption into the blood), it has been shown that metformin works largely in the colon; despite the reduced levels of absorption of metformin DR, this formulation was effective in lowering blood glucose (Buse et al, 2016). Our findings in human organoid models not only bridge the gap between murine studies and the clinical trials on diabetics but also pinpoint a mechanism via which metformin's ability to activate AMPK may serve as a therapeutic strategy to reinforce the gut barrier.

In closing, our findings underscore the importance of the SPSpathway in the gut epithelium for the maintenance of cell polarity and the epithelial barrier. By showing previously how the SPS-pathway serves as a tumor suppressive pathway (Aznar et al, 2016) and showing here how the SPS-pathway suppresses proinflammatory cytokines in the aging gut ("inflammaging"), we have linked this specialized polarity pathway polarity to epithelium-driven pathophysiologic processes. By demonstrating here that the SPS-pathway is suppressed during aging and during CRC initiation, we implicate a loss of this pathway as a bona fide pathophysiologic component of aging and an early event during oncogenesis. Insights gained also reveal the diagnostic and therapeutic potential of the SPS-pathway-its pharmacologic augmentation could be exploited as a strategy for resisting aging, CRC chemoprevention, and presumably for combating a myriad of other diseases that are characterized by chronic inflammation and endotoxemia arising from a leaky gut barrier.

\section{Materials and Methods}

\section{Reagents and antibodies}

Unless otherwise indicated, all reagents were of analytical grade and obtained from Sigma-Aldrich. Custom-designed oligos were obtained from Valuegene. Antibodies against GIV that were used in this work include rabbit serum anti-GIV coiled-coil immunoglobulin G (GIV-ccAb for immunoblotting only) (Le-Niculescu et al, 2005) and affinitypurified GIV cCAb (Cat. no. ABT80; from EMD Millipore for immunoblotting). Mouse mAbs against anti-phospho-(p; Cell signaling Technology) and total (t; Abcam) AMPK, and tubulin (Sigma-Aldrich) were purchased from commercial sources. Rabbit polyclonal antibodies against phospho-S245 GIV were generated commercially by 21st Century Biochemicals and extensively validated previously (Aznar et al, 2016). DAPI and antimouse Alexa Fluor 594-coupled goat secondary antibody for immunofluorescence were purchased from Invitrogen. Goat antirabbit and goat antimouse Alexa Fluor 680 or IRDye $800 \mathrm{~F}\left(\mathrm{ab}^{\prime}\right)_{2}$ for immunoblotting were from LI-COR Biosciences.

\section{Animal studies}

C57BL/ 6 mice of 6-8 wk age from both genders were used to isolate intestinal crypts. Animals were bred, housed, used for all the experiments, and euthanized according to the University of California San Diego Institutional Animal Care and Use Committee policies under the animal protocol number S18086. All methods were carried out in accordance with relevant guidelines and regulations and the experimental protocols were approved by institutional policies and reviewed by the licensing committee.

\section{Human subjects}

For immunohistochemical analysis of human tissue specimens, archived FFPE human colonic biopsies from healthy controls or patients with adenomas and/or carcinomas were obtained from the Gastroenterology Division, VA San Diego Healthcare System, following the protocol approved by the Human Research Protection Program Institutional Review Board (Project ID\# 1132632).

For the purpose of generating adult healthy enteroids, a fresh biopsy was prospectively collected using small forceps from healthy subjects undergoing routine colonoscopy for colon cancer screening. For all the deidentified human subjects, information including age, ethnicity, gender, previous history of disease, and medication was collected from the chart following the rules of HIPAA. Each human participant was recruited to the study following an approved human research protocol and signed a consent form approved by the Human Research Protection Program at the University of California, San Diego, to agree that the colonic specimens from their colonoscopy will be used to generate an enteroid line for functional studies.

\section{Isolation, expansion, and culture of organoids from mouse and human colons}

Intestinal crypts were isolated from the colonic tissue specimen by digesting with collagenase type I $(2 \mathrm{mg} / \mathrm{ml}$; Life Technologies Corporation) and cultured in stem cell-enriched conditioned media (CM) with WNT 3a, R-spondin, and noggin (Sato et al, 2009; Miyoshi \& Stappenbeck, 2013; Mahe et al, 2015). Briefly, the crypts after digestion with collagenase were filtered with a cell strainer and washed with the medium (DMEM/F12 with Hepes, 10\% FBS). After adding collagenase I solution containing gentamicin $(50 \mu \mathrm{g} / \mathrm{ml}$; Life Technologies Corporation) and mixing thoroughly, the plate was incubated at $37^{\circ} \mathrm{C}$ inside a $\mathrm{CO}_{2}$ incubator for $10 \mathrm{~min}$, with vigorous pipetting between incubations and monitoring constantly by light microscopy to confirm by direct observation the dislodgement of the intestinal crypts from the tissues. The collagenase was inactivated with media and filtered using a 70- $\mu$ m cell strainer over a 50 $\mathrm{ml}$ centrifuge tube. Filtered tissue was spun down at $200 \mathrm{~g}$ for $5 \mathrm{~min}$, and the media was aspirated. The epithelial units were suspended in Matrigel (basement membrane matrix; Cat. no. 356235; Corning Inc.). Cell-Matrigel suspension ( $15 \mu \mathrm{l}$ ) was placed at the center of the 24-well plate on ice and placed on the incubator upside-down for polymerization. After $10 \mathrm{~min}, 500 \mu \mathrm{l}$ of $50 \%$ CM was added. CM was prepared from L-WRN cells (CRL-3276; ATCC, from the laboratory of Thaddeus S. Stappenbeck [Miyoshi \& Stappenbeck, 2013]) with Wnt3a, R-spondin, and noggin. Y27632 (ROCK inhibitor, $10 \mu \mathrm{M}$ ) and SB431542 (an inhibitor for TGF- $\beta$ type I receptor, $10 \mu \mathrm{M}$ ) were added to the medium. For the human enteroids, media and supplements were obtained commercially (Cell Applications Inc.), and a 
proprietary cocktail was added to the above medium. The medium was changed every 2-3 d and the enteroids were expanded and frozen in liquid nitrogen.

AMPK KO enteroids were isolated from KO AMPK mice specifically deleted in the intestinal epithelial cells; these mice were generated at INSERM by crossing Villin-Cre mice with AMPK $\alpha 1^{\mathrm{ft} / \mathrm{fl}} / \alpha 2^{\mathrm{fl} / \mathrm{fl}}$ mice (manuscript in preparation). As for human studies, 4-6 healthy human individuals were used across the experiments testing their junctional integrity and response to AMPK agonists.

\section{Preparation of EDMs}

EDMs were prepared from colon spheroids as previously described (den Hartog et al, 2016; Suarez et al, 2018 Preprint). Briefly, the spheroids were trypsinized and the isolated cells were filtered, counted, resuspended in 5\% CM, and plated with Matrigel (1:40 dilution) in the apical part of $0.4-\mu \mathrm{m}$ polyester membrane transwell (Cat. no. 3470; Corning) at the density of $2 \times 10^{5}$ cell/well. In some cases, the EDMs were also differentiated for $2 \mathrm{~d}$ in advanced DMEM/ F12 media without Wnt3a but with R-spondin, noggin, B27 and N2 supplements, and $10 \mu \mathrm{M}$ ROCK inhibitor (Sato et al, 2009). As expected, this results in a marked reduction in the expression of the stemness marker Lgr5 in EDMs (Sato et al, 2009).

\section{Bacterial culture}

E. coli K12 strain DH10B, pTransSacB (PTA5105), was obtained from ATCC; the bacteria was cultured on LB agar and LB broth and used to infect EDM at moi 100.

\section{Infection of EDMs with live microbes}

Mouse and human EDMs were prepared as described in the previous section. EDMs were differentiated for $2 \mathrm{~d}$ before treatment with various chemical activators of AMPK, that is, metformin $(1 \mathrm{mM})$ and, A-769662 $(100 \mu \mathrm{M})$ for $16 \mathrm{~h}$. Cultures were then challenged with either microbial products, that is, LPS $(500 \mathrm{ng} / \mathrm{ml})$ and $\mathrm{H}_{2} \mathrm{O}_{2}(100 \mu \mathrm{M})$ or live microbes ( $E$. coli K12). On the day of infection, the medium in the basolateral part was also replaced with fresh $5 \%$ medium. Transepithelial electrical resistance (TEER) was measured using an epithelial voltohmmeter Millicell-ERS resistance meter (Millipore) before and at specific time points after the treatment $(0,4,8$, and $24 \mathrm{~h}$ ). The supernatant was collected from the basolateral and apical part of the transwell for cytokine analysis, and the cells were collected for RNA extraction followed by expression of target genes by $q R T-P C R$.

\section{Immunofluorescence}

Mouse and human EDM were fixed with cold methanol at $-20^{\circ} \mathrm{C}$ for 20 min, washed once with phosphate-buffered saline and blocked with IF buffer ( $0.1 \%$ Triton TX-100 and $2 \mathrm{mg} / \mathrm{ml}$ BSA, in PBS) for $1 \mathrm{~h}$. The samples were then incubated with primary and then secondary antibodies as described previously (Ghosh et al, 2008). Dilutions of antibodies and reagents were as follows: anti-phospho-Ser245-GIV (pS245-GIV; 1/250), anti-occludin (1/250), anti-claudin-2-1 (1/250), $\operatorname{DAPI}(1: 1,000)$, and goat antimouse Alexa Flour (488 and 594)- conjugated antibodies (1:500). Images were acquired using a Leica CTR4000 Confocal Microscope with a 63× objective. Z-stack images were obtained by imaging $\sim 4 \mathrm{~m}$-thick sections of cells in all channels. Cross-section images were obtained by automatic layering of individual slices from each Z-stack. Red-green-blue (RGB) graphic profiles were created by analyzing the distribution and intensity of pixels of these colors along a chosen line using Image I software. All individual images were processed using Image J software and assembled for presentation using Photoshop and Illustrator software (Adobe). Quantification of burst tight junctions was performed by manually counting the number of total and burst tri-cellular junctions in 8-10 randomly chosen fields in each of three independent experiments. Results are expressed as the frequency of burst TJs, and a one-way ANOVA analysis was used to determine significance.

\section{Immunoblotting}

For immunoblotting, protein samples were separated by SDS-PAGE and transferred to Polyvinylidene fluoride (PVDF) membranes (Millipore). The membranes were blocked with PBS supplemented with 5\% nonfat milk (or with 5\% BSA when probing for phosphorylated proteins) before incubation with primary antibodies. Infrared imaging with two-color detection and band densitometry quantifications were performed using a Li-Cor Odyssey imaging system exactly as performed previously (Garcia-Marcos et al, 2010, 2011a, 2011c, 2012; Ghosh et al, 2010). All Odyssey images were processed using Image software $(\mathrm{NIH})$ and assembled into figure panels using Photoshop and Illustrator software (Adobe).

\section{Immunohistochemistry (IHC)}

FFPE tissue sections of $4 \mu \mathrm{m}$ thickness were cut and placed on glass slides coated with poly-L-lysine, followed by deparaffinization and hydration. Heat-induced epitope retrieval was performed using citrate buffer ( $\mathrm{pH}$ 6) in a pressure cooker. Tissue sections were incubated with $0.3 \%$ hydrogen peroxidase for $15 \mathrm{~min}$ to block endogenous peroxidase activity, followed by incubation with primary antibodies for $30 \mathrm{~min}$ in a humidified chamber at room temperature. Antibodies used for immunostaining were anti-pS245 GIV (1:50, antirabbit antibody) and antiAMPK $\alpha 2$ (1:50, antirabbit; Abcam). Immunostaining was visualized with a labeled streptavidin-biotin using 3,3'-diaminobenzidine as a chromogen and counterstained with hematoxylin. The samples were quantitatively analyzed and scored based on the intensity of staining using the following scale; $0-3$, where $0=$ no staining, 1 = light brown, $2=$ brown, and 3 = dark brown. Data are expressed as frequency of staining score, and a chi-squared test was used to determine significance.

\section{Data reproducibility, rigor}

Each finding showcased here represents at least 3-5 independent repeats of experiments conducted on separate days. For ELISA and qPCR, results represent 2-3 technical repeats on each experiment, for a total of $n=\sim 3-5$ independent experiments. Where immunofluorescence images are shown, representative images from randomly chosen fields are presented. 


\section{Statistical analyses}

Data are expressed as the Mean \pm SEM. Statistical significance was assessed with the $t$ test. Statistical significance between datasets with three or more experimental groups was determined using one-way ANOVA including a Tukey's test for multiple comparisons. For all tests, a $P$-value of 0.05 was used as the cutoff to determine significance $\left({ }^{\star} P<\right.$ $0.05,{ }^{* *} P<0.01,{ }^{* \star *} P<0.001$, and $\left.{ }^{* * * *} P<0.0001\right)$. All experiments were repeated a least three times, and $P$-values are indicated in each figure. All statistical analyses were performed using GraphPad prism 6.1.

\section{Supplementary Information}

Supplementary Information is available at https://doi.org/10.26508/lsa. 201900481.

\section{Acknowledgements}

We thank Gordon N Gill and Marilyn G Farquhar (University of California San Diego [UCSD]) for their critical input during the preparation of the manuscript. We are thankful to Ying Dunkel, Linda Petronella Joosen, Katherine Suarez, and Eileen Lim for technical support. Funding: This work was supported by National Institutes for Health (NIH) grants DK107585 and Clinical and Translational Science Award (CTSA) grant UL1TR001442 pilot award program (to S Das); Al141630, CA238042, and CA100768 (to P Ghosh); and R00CA151673 (to D Sahoo). D Sahoo was also supported by 2017 Padres Pedal the Cause/Rady Children's Hospital Translational PEDIATRIC Cancer Research Award. S Das was also supported by a DiaComp Pilot and Feasibility award (Augusta University) and Padres Pedal the Cause/C3 2019 Moores Cancer Center Clinical and Translational Cancer Research Awards. P Ghosh and S Das were supported by $\mathrm{NIH} /$ National Center for Advancing Translational Sciences award UG3TR002968, UCOP-TrDRP grant, and by a Padres Pedal the Cause/C3 (\#PTC 2017) pilot grant award from the Moores Cancer Center. Y Mittal and L Swanson were supported by the NIH training grant (DK 0070202). Y Mittal was also supported by an NIH CTSA-funded career-development award (1TL1TR001443). SR Ibeawuchi was supported by NIH Diversity Supplement award. L Swanson was also supported by the Chancellor's Research Excellence Scholarships for Graduate Students (UCSD) and a graduate research fellowship from the microbial sciences initiative (also at UCSD).

\section{Author Contributions}

P Ghosh: conceptualization, resources, data curation, formal analysis, supervision, funding acquisition, project administration, and writing-original draft, review, and editing.

L Swanson: data curation, software, formal analysis, investigation, and methodology.

IM Sayed: data curation, formal analysis, investigation, and methodology. Y Mittal: data curation, formal analysis, investigation, and methodology. BB Lim: data curation, formal analysis, and methodology.

S-R Ibeawuchi: data curation, formal analysis, investigation, and methodology.

M Foretz: resources.

B Viollet: resources.

D Sahoo: conceptualization, resources, and software.

S Das: conceptualization, data curation, software, formal analysis, supervision, funding acquisition, investigation, methodology, project administration, and writing-original draft, review, and editing.

\section{Conflict of Interest Statement}

S Das and P Ghosh have a patent on the methodology to detect and fix leaky gut barrier by measuring the activity and augmenting the SPS-pathway. Other than that, all the authors declare that they have no conflict of interest.

\section{References}

Akbulut KG, Aktas SH, Akbulut H (2015) The role of melatonin, sirtuin2 and FoXO1 transcription factor in the aging process of colon in male rats. Biogerontology 16: 99-108. doi:10.1007/s10522-014-9540-1

Akimova T, Xiao H, Liu Y, Bhatti TR, Jiao J, Eruslanov E, Singhal S, Wang L, Han R, Zacharia K, et al (2014) Targeting sirtuin-1 alleviates experimental autoimmune colitis by induction of Foxp3+ T-regulatory cells. Mucosal Immunol 7: 1209-1220. doi:10.1038/mi.2014.10

Alam MZ, Alam Q, Kamal MA, Abuzenadah AM, Haque A (2014) A possible link of gut microbiota alteration in type 2 diabetes and Alzheimer's disease pathogenicity: An update. CNS Neurol Disord Drug Targets 13: 383-390. doi:10.2174/18715273113126660151

Aznar N, Patel A, Rohena CC, Dunkel Y, Joosen LP, Taupin V, Kufareva I, Farquhar MG, Ghosh P (2016) AMP-activated protein kinase fortifies epithelial tight junctions during energetic stress via its effector GIV/ Girdin. Elife 5: e20795. doi:10.7554/elife.20795

Barbara G (2006) Mucosal barrier defects in irritable bowel syndrome. Who left the door open? Am J Gastroenterol 101: 1295-1298. doi:10.1038/ajg2006242

Barbazan J, Dunkel Y, Li H, Nitsche U, Janssen KP, Messer K, Ghosh P (2016) Prognostic impact of modulators of $\mathrm{G}$ proteins in circulating tumor cells from patients with metastatic colorectal cancer. Sci Rep 6: 22112. doi:10.1038/srep22112

Barzilai N, Crandall JP, Kritchevsky SB, Espeland MA (2016) Metformin as a tool to target aging. Cell Metab 23: 1060-1065. doi:10.1016/j.cmet.2016.05.011

Bischoff SC, Barbara G, Buurman W, Ockhuizen T, Schulzke JD, Serino M, Tilg H, Watson A, Wells JM (2014) Intestinal permeability: A new target for disease prevention and therapy. BMC Gastroenterol 14: 189. doi:10.1186/s12876-014-0189-7

Buscarinu MC, Cerasoli B, Annibali V, Policano C, Lionetto L, Capi M, Mechelli R, Romano S, Fornasiero A, Mattei G, et al (2016) Altered intestinal permeability in patients with relapsing-remitting multiple sclerosis: A pilot study. Mult Scler 23: 442-446. doi:10.1177/1352458516652498

Buse JB, DeFronzo RA, Rosenstock J, Kim T, Burns C, Skare S, Baron A, Fineman $M$ (2016) The primary glucose-lowering effect of metformin resides in the gut, not the circulation: Results from short-term pharmacokinetic and 12-week dose-ranging studies. Diabetes Care 39: 198-205. doi:10.2337/dc15-0488

Camilleri M, Gorman H (2007) Intestinal permeability and irritable bowel syndrome. Neurogastroenterol Motil 19: 545-552. doi:10.1111/j.13652982.2007.00925.x

Clairembault T, Leclair-Visonneau L, Coron E, Bourreille A, Le Dily S, Vavasseur F, Heymann MF, Neunlist M, Derkinderen P (2015) Structural alterations of the intestinal epithelial barrier in Parkinson's disease. Acta Neuropathol Commun 3: 12. doi:10.1186/s40478-015-0196-0

Conley MN, Wong CP, Duyck KM, Hord N, Ho E, Sharpton TJ (2016) Aging and serum MCP-1 are associated with gut microbiome composition in a murine model. PeerJ 4: e1854. doi:10.7717/peerj.1854

Cool B, Zinker B, Chiou W, Kifle L, Cao N, Perham M, Dickinson R, Adler A, Gagne $G$, Iyengar $R$, et al (2006) Identification and characterization of a small molecule AMPK activator that treats key components of type 2 diabetes and the metabolic syndrome. Cell Metab 3: 403-416. doi:10.1016/j.cmet.2006.05.005 
Cunningham KE, Turner JR (2012) Myosin light chain kinase: Pulling the strings of epithelial tight junction function. Ann N Y Acad Sci 1258: 34-42. doi:10.1111/j.1749-6632.2012.06526.x

Dambroise E, Monnier L, Ruisheng L, Aguilaniu H, Joly JS, Tricoire H, Rera M (2016) Two phases of aging separated by the Smurf transition as a public path to death. Sci Rep 6: 23523. doi:10.1038/srep23523

den Hartog G, Chattopadhyay R, Ablack A, Hall EH, Butcher LD, Bhattacharyya A, Eckmann L, Harris PR, Das S, Ernst PB, et al (2016) Regulation of Rac1 and reactive oxygen species production in response to infection of gastrointestinal epithelia. PLoS Pathog 12: e1005382. doi:10.1371/ journal.ppat.1005382

Everard A, Belzer C, Geurts L, Ouwerkerk JP, Druart C, Bindels LB, Guiot Y, Derrien M, Muccioli GG, Delzenne NM, et al (2013) Cross-talk between Akkermansia muciniphila and intestinal epithelium controls diet-induced obesity. Proc Natl Acad Sci U S A 110: 9066-9071. doi:10.1073/pnas.1219451110

Foulke-Abel J, In J, Kovbasnjuk O, Zachos NC, Ettayebi K, Blutt SE, Hyser JM, Zeng XL, Crawford SE, Broughman JR, et al (2014) Human enteroids as an ex-vivo model of host-pathogen interactions in the gastrointestinal tract. Exp Biol Med (Maywood) 239: 1124-1134. doi:10.1177/1535370214529398

Franceschi C (2007) Inflammaging as a major characteristic of old people: Can it be prevented or cured? Nutr Rev 65: S173-S176. doi:10.1301/ nr.2007.dec.s173-s176

Franceschi C, Capri M, Monti D, Giunta S, Olivieri F, Sevini F, Panourgia MP, Invidia L, Celani L, Scurti M, et al (2007) Inflammaging and antiinflammaging: A systemic perspective on aging and longevity emerged from studies in humans. Mech Ageing Dev 128: 92-105. doi:10.1016/j.mad.2006.11.016

Furuse M, Izumi Y, Oda Y, Higashi T, Iwamoto N (2014) Molecular organization of tricellular tight junctions. Tissue Barriers 2: e28960. doi:10.4161/ tisb.28960

Garcia-Marcos M, Ear J, Farquhar MG, Ghosh P (2011a) A GDI (AGS3) and a GEF (GIV) regulate autophagy by balancing $G$ protein activity and growth factor signals. Mol Biol Cell 22: 673-686. doi:10.1091/mbc.e10-08-0738

Garcia-Marcos M, Ghosh P, Ear J, Farquhar MG (2010) A structural determinant that renders $G$ alpha(i) sensitive to activation by GIV/girdin is required to promote cell migration. J Biol Chem 285: 12765-12777. doi:10.1074/jbc.m109.045161

Garcia-Marcos M, Jung BH, Ear J, Cabrera B, Carethers JM, Ghosh P (2011b) Expression of GIV/Girdin, a metastasis-related protein, predicts patient survival in colon cancer. FASEB J 25: 590-599. doi:10.1096/fj.10-167304

Garcia-Marcos M, Kietrsunthorn PS, Pavlova Y, Adia MA, Ghosh P, Farquhar MG (2012) Functional characterization of the guanine nucleotide exchange factor (GEF) motif of GIV protein reveals a threshold effect in signaling. Proc Natl Acad Sci U S A 109: 1961-1966. doi:10.1073/ pnas. 1120538109

Garcia-Marcos M, Kietrsunthorn PS, Wang H, Ghosh P, Farquhar MG (2011c) G Protein binding sites on Calnuc (nucleobindin 1) and NUCB2 (nucleobindin 2) define a new class of G(alpha)i-regulatory motifs. J Biol Chem 286: 28138-28149. doi:10.1074/jbc.m110.204099

Ghosh P (2017) The stress polarity pathway: AMPK “GIV"-es protection against metabolic insults. Aging (Albany NY) 9: 303-314. doi:10.18632/ aging.101179

Ghosh P (2015) Heterotrimeric G proteins as emerging targets for network based therapy in cancer: End of a long futile campaign striking heads of a Hydra. Aging (Albany NY) 7: 469-474. doi:10.18632/aging.100781

Ghosh P, Beas AO, Bornheimer SJ, Garcia-Marcos M, Forry EP, Johannson C, Ear J, Jung BH, Cabrera B, Carethers JM, et al (2010) A G\{alpha\}i-GIV molecular complex binds epidermal growth factor receptor and determines whether cells migrate or proliferate. Mol Biol Cell 21: 2338-2354. doi:10.1091/mbc.e10-01-0028
Ghosh P, Garcia-Marcos M, Bornheimer SJ, Farquhar MG (2008) Activation of Galphai3 triggers cell migration via regulation of GIV. J Cell Biol 182: 381-393. doi:10.1083/jcb.200712066

Ghosh P, Tie J, Muranyi A, Singh S, Brunhoeber P, Leith K, Bowermaster R, Liao Z, Zhu Y, LaFleur B, et al (2016) Girdin (GIV) expression as a prognostic marker of recurrence in mismatch repair-proficient stage II colon cancer. Clin Cancer Res 22: 3488-3498. doi:10.1158/1078-0432.ccr-15-2290

Giunta S (2006) Is inflammaging an auto[innate]immunity subclinical syndrome? Immun Ageing 3: 12. doi:10.1186/1742-4933-3-12

Goto M (2008) Inflammaging (inflammation + aging): A driving force for human aging based on an evolutionarily antagonistic pleiotropy theory? Biosci Trends 2: 218-230.

Igarashi M, Miura M, Williams E, Jaksch F, Kadowaki T, Yamauchi T, Guarente L (2019) NAD(+) supplementation rejuvenates aged gut adult stem cells. Aging Cell 18: e12935. doi:10.1111/acel.12935

Jun BY, Kim SW, Jung CK, Cho YK, Lee IS, Choi MG, Choi KY, Oh ST (2013) Expression of girdin in human colorectal cancer and its association with tumor progression. Dis Colon Rectum 56: 51-57. doi:10.1097/ dcr.0b013e31826b9b7e

Kaeberlein M, McVey M, Guarente L (1999) The SIR2/3/4 complex and SIR2 alone promote longevity in Saccharomyces cerevisiae by two different mechanisms. Genes Dev 13: 2570-2580. doi:10.1101/ gad.13.19.2570

Katz D, Hollander D, Said HM, Dadufalza V (1987) Aging-associated increase in intestinal permeability to polyethylene glycol 900 . Dig Dis Sci 32: 285-288. doi:10.1007/bf01297055

Le-Niculescu H, Niesman I, Fischer T, DeVries L, Farquhar MG (2005) Identification and characterization of GIV, a novel Galpha i/sinteracting protein found on COPI, endoplasmic reticulum-Golgi transport vesicles. J Biol Chem 280: 22012-22020. doi:10.1074/ jbc.m501833200

Lee JH, Koh H, Kim M, Kim Y, Lee SY, Karess RE, Lee SH, Shong M, Kim JM, Kim J, et al (2007) Energy-dependent regulation of cell structure by AMPactivated protein kinase. Nature 447: 1017-1020. doi:10.1038/ nature05828

Lee SY, Lee SH, Yang EJ, Kim EK, Kim JK, Shin DY, Cho ML (2015) Metformin ameliorates inflammatory bowel disease by suppression of the STAT3 signaling pathway and regulation of the between Th17/Treg balance. PLOS One 10: e0135858. doi:10.1371/journal.pone.0135858

Liu F, Bu HF, Geng H, De Plaen IG, Gao C, Wang P, Wang X, Kurowski JA, Yang H, Qian J, et al (2017a) Sirtuin-6 preserves R-spondin-1 expression and increases resistance of intestinal epithelium to injury in mice. Mol Med 23: 272-284. doi:10.2119/molmed.2017.00085

Liu F, Bu HF, Geng H, De Plaen IG, Gao C, Wang P, Wang X, Kurowski JA, Yang H, Qian J, et al (2017b) Sirtuin-6 preserves R-spondin-1 expression and increases resistance of intestinal epithelium to injury in mice. Mol Med 23: 272-284. doi:10.2119/molmed.2017.00085

Lo Sasso G, Menzies KJ, Mottis A, Piersigilli A, Perino A, Yamamoto H, Schoonjans K, Auwerx J (2014) SIRT2 deficiency modulates macrophage polarization and susceptibility to experimental colitis. PLoS One 9: e103573. doi:10.1371/journal.pone.0102495

Lu J, Zhang L, Zhou H, Du Z, Zhang G (2018) Silencing of Girdin suppresses the malignant behavior of colorectal carcinoma cells. Oncol Rep 40: 887-894. doi:10.3892/or.2018.6511

Mahe MM, Sundaram N, Watson CL, Shroyer NF, Helmrath MA (2015) Establishment of human epithelial enteroids and colonoids from whole tissue and biopsy. J Vis Exp 52483. doi:10.3791/52483

Manabe I (2017) Inflammaging and age-associated diseases. Nihon Ronen Igakkai Zasshi 54: 105-113. doi:10.3143/geriatrics.54.105

Martin-Belmonte F, Perez-Moreno M (2012) Epithelial cell polarity, stem cells and cancer. Nat Rev Cancer 12: 23-38. doi:10.1038/nrc3169 
Minciullo PL, Catalano A, Mandraffino G, Casciaro M, Crucitti A, Maltese G, Morabito N, Lasco A, Gangemi S, Basile G (2016) Inflammaging and anti-inflammaging: The role of cytokines in extreme longevity. Arch Immunol Ther Exp (Warsz) 64: 111-126. doi:10.1007/s00005-015-0377-3

Miyoshi H, Stappenbeck TS (2013) In vitro expansion and genetic modification of gastrointestinal stem cells in spheroid culture. Nat Protoc 8: 2471-2482. doi:10.1038/nprot.2013.153

Noel G, Baetz NW, Staab JF, Donowitz M, Kovbasnjuk O, Pasetti MF, Zachos NC (2017) A primary human macrophage-enteroid co-culture model to investigate mucosal gut physiology and host-pathogen interactions. Sci Rep 7: 45270. doi:10.1038/srep46790

Nouri M, Bredberg A, Westrom B, Lavasani S (2014) Intestinal barrier dysfunction develops at the onset of experimental autoimmune encephalomyelitis, and can be induced by adoptive transfer of autoreactive T cells. PLoS One 9: e106335. doi:10.1371/journal.pone.0106335

Rera M, Clark RI, Walker DW (2012) Intestinal barrier dysfunction links metabolic and inflammatory markers of aging to death in Drosophila. Proc Natl Acad Sci U S A 109: 21528-21533. doi:10.1073/pnas.1215849110

Rogina B, Helfand SL (2004) Sir2 mediates longevity in the fly through a pathway related to calorie restriction. Proc Natl Acad Sci U S A 101: 15998-16003. doi:10.1073/pnas.0404184101

Samsam M, Ahangari R, Naser SA (2014) Pathophysiology of autism spectrum disorders: Revisiting gastrointestinal involvement and immune imbalance. World J Gastroenterol 20: 9942-9951. doi:10.3748/wjg.v20.i29.9942

Sandek A, Bauditz J, Swidsinski A, Buhner S, Weber-Eibel J, von Haehling S, Schroedl W, Karhausen T, Doehner W, Rauchhaus M, et al (2007) Altered intestinal function in patients with chronic heart failure. J Am Coll Cardiol 50: 1561-1569. doi:10.1016/j.jacc.2007.07.016

Sandek A, Bjarnason I, Volk HD, Crane R, Meddings JB, Niebauer J, Kalra PR, Buhner S, Herrmann R, Springer J, et al (2012) Studies on bacterial endotoxin and intestinal absorption function in patients with chronic heart failure. Int J Cardiol 157: 80-85. doi:10.1016/j.ijcard.2010.12.016

Sandek A, Rauchhaus M, Anker SD, von Haehling S (2008) The emerging role of the gut in chronic heart failure. Curr Opin Clin Nutr Metab Care 11: 632-639. doi:10.1097/mco.0b013e32830a4c6e

Sato T, Vries RG, Snippert HJ, van de Wetering M, Barker N, Stange DE, van Es JH, Abo A, Kujala P, Peters PJ, et al (2009) Single Lgr5 stem cells build crypt-villus structures in vitro without a mesenchymal niche. Nature 459: 262-265. doi:10.1038/nature07935

Shackelford DB, Shaw RJ (2009) The LKB1-AMPK pathway: Metabolism and growth control in tumour suppression. Nat Rev Cancer 9: 563-575. doi:10.1038/nrc2676

Shaw RJ, Lamia KA, Vasquez D, Koo SH, Bardeesy N, Depinho RA, Montminy M, Cantley LC (2005) The kinase LKB1 mediates glucose homeostasis in liver and therapeutic effects of metformin. Science 310: 1642-1646. doi:10.1126/science. 1120781

St Johnston D, Ahringer J (2010) Cell polarity in eggs and epithelia: Parallels and diversity. Cell 141: 757-774. doi:10.1016/j.cell.2010.05.011

St Johnston D, Sanson B (2011) Epithelial polarity and morphogenesis. Curr Opin Cell Biol 23: 540-546. doi:10.1016/j.ceb.2011.07.005

Suarez K, Lim E, Singh S, Pereira M, Joosen LP, Ibeawuchi S-R, Dunkel Y, Mittal Y, Ho SB, Chattopadhyay R, et al (2018) Dysregulation of the engulfment pathway in the gut fuels Inflammatory Bowel Disease. BioRxiv 280172. doi:10.1101/280172 Preprint posted March 12, 2018.

Thevaranjan N, Puchta A, Schulz C, Naidoo A, Szamosi JC, Verschoor CP, Loukov D, Schenck LP, Jury J, Foley KP, et al (2017) Age-associated microbial dysbiosis promotes intestinal permeability, systemic inflammation, and macrophage dysfunction. Cell Host Microbe 21: 455-466.e4. doi:10.1016/j.chom.2017.03.002
Tissenbaum HA, Guarente L (2001) Increased dosage of a sir-2 gene extends lifespan in Caenorhabditis elegans. Nature 410: 227-230. doi:10.1038/ 35065638

Tran L, Greenwood-Van Meerveld B (2013) Age-associated remodeling of the intestinal epithelial barrier. J Gerontol A Biol Sci Med Sci 68: 1045-1056. doi:10.1093/gerona/glt106

Tricoire H, Rera M (2015) A new, discontinuous 2 phases of aging model: Lessons from Drosophila melanogaster. PLoS One 10: e0141920. doi:10.1371/journal.pone.0141920

Tsukita S, Yamazaki Y, Katsuno T, Tamura A, Tsukita S (2008) Tight junctionbased epithelial microenvironment and cell proliferation. Oncogene 27: 6930-6938. doi:10.1038/onc.2008.344

Valentini L, Ramminger S, Haas V, Postrach E, Werich M, Fischer A, Koller M, Swidsinski A, Bereswill S, Lochs H, et al (2014) Small intestinal permeability in older adults. Physiol Rep 2: e00281. doi:10.1002/ phy2.281

van De Sande MM, van Buul VJ, Brouns FJ (2014) Autism and nutrition: The role of the gut-brain axis. Nutr Res Rev 27: 199-214. doi:10.1017/ s0954422414000110

Wellman AS, Metukuri MR, Kazgan N, Xu X, Xu Q, Ren NSX, Czopik A, Shanahan MT, Kang A, Chen W, et al (2017) Intestinal epithelial sirtuin 1 regulates intestinal inflammation during aging in mice by altering the intestinal microbiota. Gastroenterology 153: 772-786. doi:10.1053/ j.gastro.2017.05.022

Wodarz A, Nathke I (2007) Cell polarity in development and cancer. Nat Cell Biol 9: 1016-1024. doi:10.1038/ncb433

Xiao B, Sanders MJ, Carmena D, Bright NJ, Haire LF, Underwood E, Patel BR Heath RB, Walker PA, Hallen S, et al (2013) Structural basis of AMPK regulation by small molecule activators. Nat Commun 4: 3017. doi:10.1038/ncomms4017

Xue Y, Zhang H, Sun X, Zhu MJ (2016) Metformin improves ileal epithelial barrier function in interleukin-10 deficient mice. PLoS One 11: e0168670. doi:10.1371/journal.pone. 0168670

Yacyshyn B, Meddings J, Sadowski D, Bowen-Yacyshyn MB (1996) Multiple sclerosis patients have peripheral blood CD45RO+ B cells and increased intestinal permeability. Dig Dis Sci 41: 2493-2498. doi:10.1007/bf02100148

Yu H, Hasan NM, In JG, Estes MK, Kovbasnjuk O, Zachos NC, Donowitz M (2017) The contributions of human mini-intestines to the study of intestinal physiology and pathophysiology. Annu Rev Physiol 79: 291-312. doi:10.1146/annurev-physiol-021115-105211

Zhang L, Li J, Young LH, Caplan MJ (2006) AMP-activated protein kinase regulates the assembly of epithelial tight junctions. Proc Natl Acad Sci U S A 103: 17272-17277. doi:10.1073/pnas.0608531103

Zhang Y, Wang XL, Zhou M, Kang C, Lang HD, Chen MT, Hui SC, Wang B, Mi MT (2018) Crosstalk between gut microbiota and Sirtuin-3 in colonic inflammation and tumorigenesis. Exp Mol Med 50: 21. doi:10.1038/ s12276-017-0002-0

Zheng B, Cantley LC (2007) Regulation of epithelial tight junction assembly and disassembly by AMP-activated protein kinase. Proc Natl Acad Sci U S A 104: 819-822. doi:10.1073/pnas.0610157104

Zhou ZY, Ren LW, Zhan P, Yang HY, Chai DD, Yu ZW (2016) Metformin exerts glucose-lowering action in high-fat fed mice via attenuating endotoxemia and enhancing insulin signaling. Acta Pharmacol $\operatorname{Sin} 37$ 1063-1075. doi:10.1038/aps.2016.21

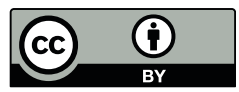

License: This article is available under a Creative Commons License (Attribution 4.0 International, as described at https://creativecommons.org/ licenses/by/4.0/). 